\title{
Online Virtual Machine Migration for Renewable Energy Usage „Maximization in Geographically Distributed Cloud Data Centers
} 9

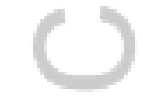

\author{
Atefeh Khosravi* Adel Nadjaran Toosi and Rajkumar Buyya
}

Cloud Computing and Distributed Systems (CLOUDS) Laboratory, Department of Computing and Information Systems,

The University of Melbourne, Australia.

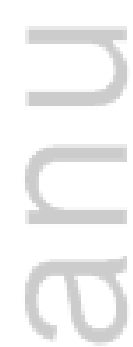

Energy consumption and its associated costs represent a huge part of cloud providers' operational costs. In this paper, we explore how much energy cost savings can be made knowing the future level of renewable energy (solar/wind) available in data centers. Since renewable energy sources have intermittent nature, we take advantage of migrating Virtual Machines (VMs) to the nearby data centers with excess renewable energy. In particular, we first devise an optimal offline algorithm with full future knowledge of renewable level in the system. Since in practice, accessing long-term and exact future knowledge of renewable energy level is not feasible, we propose two online deterministic algorithms, one with no future knowledge called deterministic and one with limited knowledge of the future renewable availability called future-aware. We show that the deterministic and future-aware algorithms are $1+1 / s$ and $1+1 / s-\omega / s \cdot T_{m}$ competitive in comparison to the optimal offline algorithm, respectively, where $s$ is the network to the brown energy cost, $\omega$ is the look-ahead window-size, and $T_{m}$ is the migration time. The effectiveness of the proposed algorithms is analyzed through extensive simulation studies using real-world traces of meteorological data and Google cluster workload. Copyright (c) 2010 John Wiley \& Sons, Ltd.

This is the author manuscript accepted for publication and has undergone full peer review but has noceived been through the copyediting, typesetting, pagination and proofreading process, which may lead to differences between this version and the Version of Record. Please cite this article

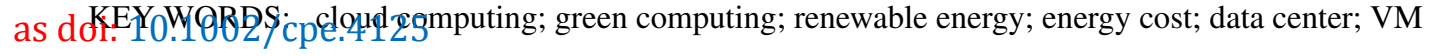
migration; online algorithms 


\section{INTRODUCTION}

Cloud computing is a paradigm focused on the realization and long held dream of delivering computing as a utility [1]. Nowadays, the number of individuals and organizations shifting their workload to cloud data centers is growing more than ever. Cloud services are delivered via data center sites each containing tens of thousands of servers, which are distributed across geographical locations. The geographical diversity of computing resources brings several benefits, such as high availability, effective disaster recovery, uniform access to users in different regions, and access to different energy sources.

Data centers as the heart of a cloud computing system are energy intensive. This is due to the high power required to run the IT equipment, power, and cooling infrastructure [2]. Based on the report by Koomey [3], data centers were responsible for $1 \%$ of the world's total energy consumption in the year 2005, equivalent to 152 billion kilowatt-hours $(\mathrm{kWh})$ that has been almost doubled from the year 2000. Besides the high energy consumption of data centers, the cost associated with the energy is a big concern as well. According to Hamilton [4], the energy costs are estimated to be around $42 \%$ of the data center's operational costs. Furthermore, the issue of high energy consumption by data centers makes them responsible for $2 \%$ of the world's total $\mathrm{CO}_{2}$ emission [5].

To overcome the problem of high energy consumption that leads to high energy costs for the cloud provider and environmental concerns due to the high $\mathrm{CO}_{2}$ emission of energy sources, there are two possible solutions: 1) improving the data center's efficiency or 2) replacing the brown energy sources with clean energy sources. By making data centers energy efficient and aware of energy sources, cloud providers are able to reduce their costs significantly [6]. Recently, large IT companies started to build their own on-site renewable energy sources, such as Facebook's solar-powered data center in Oregon [7], its newly build wind-powered data center in Texas [8], Amazon [9], Apple [10],

* Correspondence to: Atefeh Khosravi, CLOUDS Lab, Department of Computing and Information Systems, The University of Melbourne, Melbourne, Australia.E-mail: atefehk@student.unimelb.edu.au 
Google [11], and Microsoft [12] renewable energy farms. To this end, we consider access to onsite renewable energy sources ${ }^{\dagger}$, which is becoming popular for modern data center sites. However, due to the intermittent nature of renewable energy sources, these data centers consider access to off-site electrical grid (also known as brown energy) to power their infrastructure in the absence of renewables. The on-site energy sources considered are solar and wind, the two fastest growing renewables. As discussed earlier, these energy sources are not available all the time. Solar energy is only available during the day and it has its peak during noon, while wind energy fluctuates during the day and does not follow any particular pattern. Yet, cloud providers try to minimize their energy cost through maximizing on-site renewable energy usage. However, maximizing renewable energy usage in one data center site is challenging, because of intermittent and the limited nature of solar and wind energy. One solution to achieve this goal is to migrate the load (VMs) from one data center without currently available renewable energies to a data center with excess renewable energy. Moreover, migrating the VMs requires the knowledge of the time that migration should take place to avoid brown energy usage.

In this paper, we are motivated by the following question: "with limited or no priori knowledge of the future level of renewable energies, when should VM migration take place such that the energy cost is minimized and accordingly the overall renewable energy consumption is maximized?" For this, we study cost-minimizing VM migration algorithms targeting a cloud provider with distributed data center sites within a region ${ }^{\ddagger}$ with access to disparate renewable energy sources. We model the cost minimizing VM migration problem, and determine the cost of offline algorithm, as well as the competitive ratio for the optimal online deterministic algorithm. Moreover, we enhance the online algorithm by adding limited future knowledge of available renewable energy in the system. We evaluate the proposed algorithms through extensive simulation using CloudSim toolkit [14], traces

$\lceil$ Renewable and green energy sources are used interchangeably in the paper.

¥ A region is a separate geographic area with multiple and isolated locations known as availability zones connected through dedicated low latency links. This is the same definition used by Amazon EC2 architecture [13]. 
of wind and solar energy undertaken by the National Renewable Energy Laboratory (NREL) [15], and real-world workload traces from Google [16].

The main contributions of this paper are:

Formulation of the offline cost optimization problem for VM migration, across geographically distributed cloud data centers, with respect to the availability of renewable energy.

- Proof and competitive ratio analysis of the optimal online deterministic algorithm with no future knowledge against the optimal offline algorithm.

- Design of an online VM migration solution with limited future knowledge regarding the solar/wind power availability.

- Evaluation of the proposed algorithms through extensive simulations using real-world renewable energy (solar and wind) traces and workload traces of a Google cluster.

The remainder of the paper is organized as follows. The next section discusses the related work. The system model and cost optimization problem are formalized in Section 3. Section 4 presents the optimal offline solution followed, in Section 5, by introducing the online deterministic and futureaware online algorithms. Evaluation results are presented in Section 6 and Section 7 concludes the paper.

\section{RELATED WORK}

The context of energy-efficient resource management has gained considerable attention over the last few years. Moreover, along with the objective of energy consumption optimization, the problem of reducing carbon footprint has been an ongoing research due to environmental concerns, rise in global warming, social and governmental pressure (impose of carbon tax), and more importantly increase in the usage of renewable energy sources to power data center sites by cloud providers [17]. Most of the early works on energy efficiency focus on a single server and intra-data center optimization techniques, such as CPU DVFS (dynamic voltage and frequency 
scaling) [18], virtualization [19], VM migration and consolidation for switching off idle servers and power management [20, 21]. An extensive taxonomy and survey by Beloglazov et al. [22] discusses different techniques on energy-efficient data centers. Similar to our work, Beloglazov and Buyya [23] formulated cost for the single VM migration and dynamic VM consolidation problems within a single data center environment. They conducted competitive-ratio analysis to characterize the performance of optimal online algorithms against the optimal offline competitor. On the contrary, we focus on energy cost minimization by applying VM migration between data center sites considering access to renewable energy sources and limiting brown energy usage. While they consider Service Level Agreement (SLA) violation cost due to server over-subscription, we consider inter-data center network cost and additional brown energy usage.

Following the high energy consumption by data centers, increase in their operational costs, and the issue of carbon footprint encouraged cloud providers to have their own on-site renewable energy sources and power their data centers completely or partially through clean energy sources [7, 24]. Kong and Liu [25] investigated research works towards green-energy-aware power management for single and multi data centers. Recently, there has been a large body of literature considering reducing energy costs targeting inter-data center sites. They achieve this goal either by considering spatial (different electricity prices in different geographical locations) or temporal changes (different electricity prices during different times of the day) of the electricity derived from off-site grid or by maximizing renewable energy usage, which leads to minimizing brown energy consumption as well.

One of the earliest studies that targets reducing the costs associated with brown energy consumption is done by Le et al. [26]. They consider the amount of load each data center can accommodate based on its electricity price and energy source, whether it is brown or green energy and within a specific time period and budget. A similar work by Liu et al. [27] considers geographical load balancing to minimize brown energy consumption through an optimal mix of renewable energy sources (solar and wind) as well as storage of these renewables in data centers. An extension to that work has been done by Lin et al. [28] to explore the optimal combination of brown and green (solar/wind) energy sources aiming a net-zero brown energy system. To tackle the 
same problem, Toosi and Buyya [29] proposed a fuzzy logic-based load balancing algorithm that needs no knowledge of future. All these works consider routing of incoming load to the data centers based on their initial renewable/brown state by the time of users' requests submission. Whilst, we consider VM migration between data center sites, due to the limited and intermittent nature of renewable energy sources.

Rao et al. [30] aimed at minimizing total cost by considering electricity pricing data to route delay-constraint applications. Ren et al. [31] proposed online algorithms to route jobs to the data centers with low electricity prices or suspend jobs and resume them later, if necessary. Buchbinder et al. [32] has the same objective of reducing energy cost for a cloud provider. They take advantage of dynamic electricity pricing to migrate running batch jobs to the data center with lower electricity price. Comparatively, we focus on VM migration and taking advantage of available renewable energy sources in data centers.

Towards reducing energy cost and limiting brown energy consumption, Chen et al. [33] proposed scheduling algorithms to forward incoming jobs to the data centers considering energy source at the data center and requests' deadline to process the incoming requests for further execution. Celesti et al. [34] proposed a framework to allocate VM requests to the data centers with the highest level of solar energy and lowest cost. Le et al. [35] used the same idea of assigning incoming requests to the data center considering green energy sources and electricity price in order to minimize brown energy consumption. Luo et al. [36] leverage both the spatial and temporal variation of electricity price to route the incoming requests between geographically distributed data centers targeting energy cost minimization.

The comparison of the existing literature with our proposed work is summarized in Table I. Our work is different from the discussed studies, since we consider VM migration between data centers to maximize renewable energy (solar/wind) consumption. The targeted system here has several data centers located within a region (geographically near locations). We analyze the VM migration problem by calculating the optimal offline cost and computing the competitive ratio for an online 
Table I. Comparison of proposed work with existing literature.

\begin{tabular}{|c|c|c|c|c|c|}
\hline & $\begin{array}{l}\text { Energy cost } \\
\text { minimization }\end{array}$ & $\begin{array}{l}\text { Brown energy } \\
\text { minimization }\end{array}$ & $\begin{array}{c}\text { Renewable } \\
\text { energy } \\
\text { maximization }\end{array}$ & Migration & $\begin{array}{c}\text { Competitive- } \\
\text { ratio } \\
\text { analysis }\end{array}$ \\
\hline Le et al. [26] & $\checkmark$ & $\checkmark$ & $\checkmark$ & & \\
\hline Liu et al. [27] & & $\checkmark$ & $\checkmark$ & & \\
\hline Lin et al. [28] & $\checkmark$ & $\checkmark$ & $\checkmark$ & & $\checkmark$ \\
\hline Rao et al. [30] & $\checkmark$ & & & & \\
\hline Le et al. [37] & $\checkmark$ & $\checkmark$ & $\checkmark$ & & \\
\hline Ren et al. [31] & $\checkmark$ & & & & $\checkmark$ \\
\hline Buchbinder et al. [32] & $\checkmark$ & & & $\checkmark$ & $\checkmark$ \\
\hline Chen et al. [33] & & $\checkmark$ & $\checkmark$ & & \\
\hline Celesti et al. [34] & $\checkmark$ & $\checkmark$ & $\checkmark$ & & \\
\hline Le et al. [35] & $\checkmark$ & $\checkmark$ & $\checkmark$ & & \\
\hline Luo et al. [36] & $\checkmark$ & & & & \\
\hline Toosi and Buyya [29] & $\checkmark$ & $\checkmark$ & $\checkmark$ & & \\
\hline Our work & $\checkmark$ & $\checkmark$ & $\checkmark$ & $\checkmark$ & $\checkmark$ \\
\hline
\end{tabular}

deterministic algorithm, without any future knowledge of renewable energy level, and a futureaware online algorithm with a look-ahead window and limited knowledge, up to a window-size, of future level of solar and wind energy.

\section{SYSTEM SPECIFICATION AND PROBLEM DEFINITION}

\subsection{System Model}

The targeted system in this paper is an IaaS cloud provider offering virtual machine (VM) resources to its clients similar to Elastic Compute Cloud (EC2) service by Amazon Web Services [13]. The cloud provider, as shown in Figure 1, consists of several geographically distributed regions. Each region is isolated from other regions and consists of several availability zones. The availability 


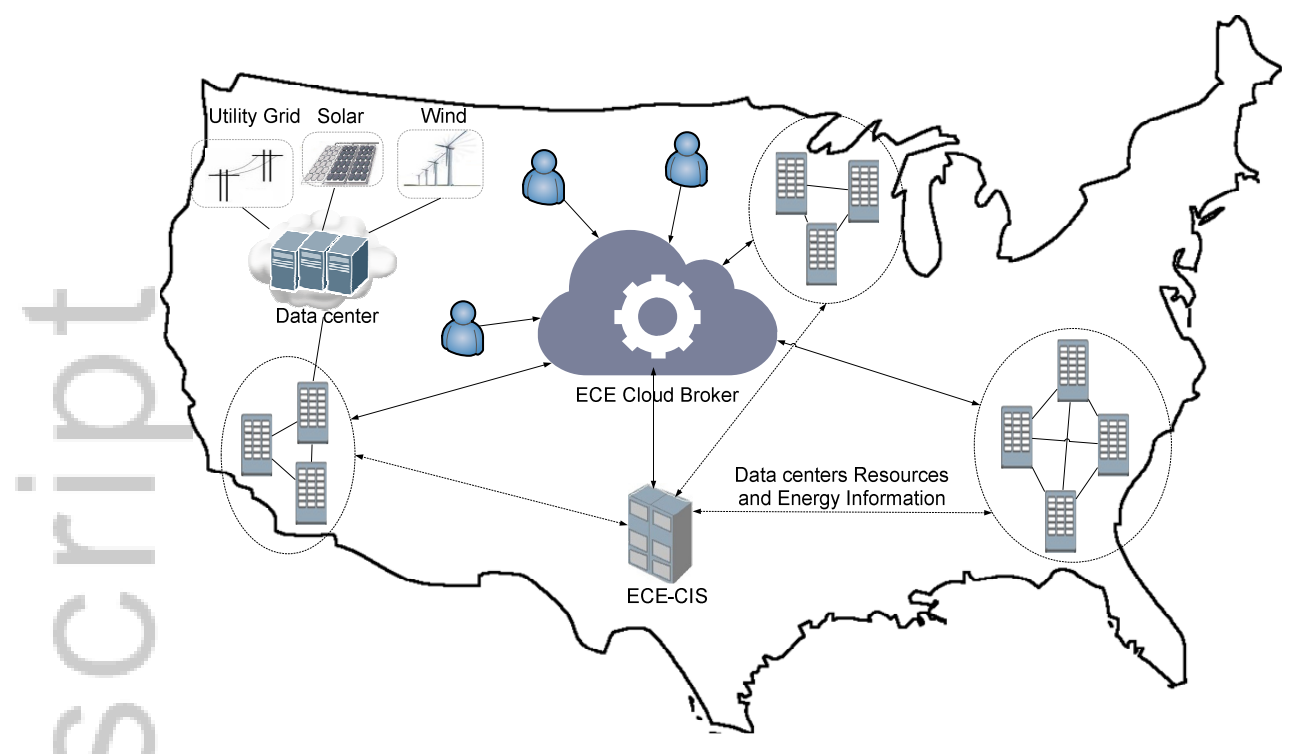

Figure 1. System model.

zones in the regions are data centers connected through low latency links. Hereafter, whenever we talk about data centers, we refer to the availability zones within one region. We only consider VM migration between data center sites belonging to the same region, as the network cost and delay associated with that is acceptable [13]. To the best of knowledge, we are the first considering VM migration between cloud data centers to maximize renewable energy utilization.

A cloud user (hereafter called user) at the time of submitting a VM provisioning request can choose the availability zone he/she wants to run the VM in or leaves the availability zone selection up to the cloud provider. Users submit VM provisioning requests through a cloud interface called a cloud broker (hereafter called broker). This connects them to the cloud provider and enables the users to monitor and follow the status of their requests. Broker, as discussed in our previous work [38], is a major component of the provider. It is responsible for receiving VM requests, performing the VM placement and migrating the currently running VM to another data center, in case of failure, maximizing renewable energy usage, or any other purpose. The information needed by the broker to function is provided by the directory called Energy and Carbon Efficient Cloud Information Service (ECE-CIS). Data centers register themselves at the ECE-CIS and keep it updated regarding any changes in their current state. The information sent by data centers to 
the ECE-CIS include: available physical resources, data center's PUE, energy source(s), amount of available renewable energy, carbon footprint rate, and physical servers' current utilization. Note that PUE stands for power usage effectiveness and is a metric coined by the Green Grid consortium [39] to represent a data center's energy efficiency. PUE is the ratio of total power consumed by the data center to its power consumed by IT devices.

As shown in Figure 1, data centers might use their own on-site renewable energy sources to power their infrastructure and servers in addition to the electricity delivered from off-site grid. The off-site grid energy is usually derived from polluting sources, known as brown energy, so cloud providers are deploying their own on-site renewable energy sources with the aim of cost saving and social impact. Two renewable energy sources considered in this work are solar photovoltaic (PV) and wind, since they are the most common and the fastest growing ones. Solar energy, as can be seen in Figure 4a, has a raised cosine distribution during the day, therefore its peak energy level varies by change in time zone for different locations. In contrast, wind energy does not have a clear predictable pattern, as shown in Figure 4b. Having these two renewable sources in a data center provides access to clean energy to run requests during different times of the day.

\subsection{Preliminaries}

We consider a cloud provider with a set of $n$ data center sites, shown as $\mathcal{D}=\left\{d_{1}, d_{2}, \ldots, d_{n}\right\}$, distributed in a geographical region. Each data center is referred to as an availability zone which consists of a set of $h$ servers/hosts shown as $\mathcal{S}=\left\{s_{1}, s_{2}, . ., s_{h}\right\}$. The list of all the symbols used in this paper are given in Table II.

Total Cost. The total cost of energy, Equation (1), is the cost of energy used to run/execute VMs in the data center and the extra cost.

$$
C_{\text {total }}=C_{\text {execution }}+C_{\text {extra }}
$$

Extra Cost. Extra cost, Equation (2), is associated with the energy used to migrate VMs between data center sites and the additional brown energy usage in the source data center while VM migration 
Table II. Description of symbols.

\begin{tabular}{|c|c|c|c|}
\hline Symbol & Description & Symbol & Description \\
\hline $\mathcal{D}$ & Set of data center sites & $n$ & Number of data center sites \\
\hline $\mathcal{S}$ & $\begin{array}{l}\text { Set of physical servers (hosts) in a } \\
\text { data center }\end{array}$ & $h$ & Number of physical servers (hosts) \\
\hline$C_{\text {execution }}$ & Energy cost to execute the VMs & $C_{\text {extra }}$ & Extra cost due to VM migration \\
\hline$C_{m i g r}$ & Energy cost to migrate the VMs & $C_{\text {addBrown }}$ & $\begin{array}{l}\text { Additional brown energy consumption } \\
\text { at the source data center during VM } \\
\text { migration }\end{array}$ \\
\hline$C_{s}$ & Cost of server energy consumption & $C_{o}$ & Cost of overhead energy consumption \\
\hline$c_{n}$ & $\begin{array}{l}\text { Cost of network to migrate the VM per } \\
\text { unit time }\end{array}$ & $c_{b}$ & Cost of brown energy per unit time \\
\hline$p_{r}$ & Price of renewable energy per unit usage & $p_{b}$ & Price of brown energy per unit usage \\
\hline$E_{r}$ & $\begin{array}{l}\text { Servers total renewable energy } \\
\text { consumption }\end{array}$ & $E_{b}$ & Servers total brown energy consumption \\
\hline$t_{m}$ & $\begin{array}{l}\text { Start time of VM migration at the source } \\
\text { data center }\end{array}$ & $T_{m}$ & Duration to migrate the VM \\
\hline$t_{b}$ & $\begin{array}{l}\text { Start time of brown energy consumption } \\
\text { at the source data center }\end{array}$ & $T_{b}$ & $\begin{array}{l}\text { Duration of brown energy usage during } \\
\text { VM migration at the source data center }\end{array}$ \\
\hline
\end{tabular}

takes place.

$$
C_{\text {extra }}=C_{\text {migration }}+C_{\text {addBrown }} .
$$

The aforementioned costs (execution, migration, and additional brown) can be detailed as follows:

Execution Cost. Execution cost is the energy cost to run VMs in the data center and is shown in Equation (3). The energy cost to run VMs consists of server $\left(C_{s}\right)$ and overhead $\left(C_{o}\right)$ costs imposed due to running VMs within a data center.

$$
C_{\text {execution }}=C_{s}+C_{o} .
$$


In order to calculate overhead energy, we use $P U E$ that is equal to the total energy goes to a data center divided by the total energy consumed by IT devices and is computed as:

$$
P U E=\frac{C_{s}+C_{o}}{C_{s}}
$$

As a result,

$$
C_{\text {execution }}=C_{s} \times P U E \text {. }
$$

Server Cost. Servers host the incoming workload and based on their configuration are capable to accommodate different number of VMs. The cost of servers $C_{s}$ is computed as follows:

$$
C_{s}=p_{r} \times E_{r}+p_{b} \times E_{b},
$$

where $E_{r}$ and $E_{b}$ are the energy consumption of servers using renewable and brown energy sources and $p_{r}$ and $p_{b}$ are the related prices, respectively.

The energy consumption of servers is the product of the power consumption of servers and the time period they have been working under that power. The power consumption depends on several hardware resources including CPU, memory, and disks [40]. According to Blackburn and Grid [41], the total power consumed by a server is determined by the incoming load to that server, which is shown as CPU utilization. The relationship between the server power consumption and CPU utilization can be a constant, cubic, or quadratic [42].

Migration Cost. Migration cost is part of the extra cost and is the energy consumed by the network to migrate the VMs between data center sites. Live migration of VMs requires relocating the VMs and placing them in their new destination [43]. The cost due to transferring the VMs is proportional to the VM size and the number of bytes that need to be transferred between data center sites, similar to AWS EC2 pricing [13]. For the sake of simplicity, we limit the migration cost to a specific type of VM with a constant network cost $c_{n}$ per unit time for the live migration and $T_{m}$ is the time required to perform and complete the migration. Equation (7) represents the migration cost.

$$
C_{\text {migration }}=c_{n} \times T_{m}
$$


Additional Brown Energy Cost. This part represents the penalty of brown energy consumption while VM migration takes place at the source data center. As mentioned earlier, we consider two different types of energy sources: brown and renewable. The renewable energy is drawn from on-site solar and wind power generators. Therefore, there is a one-time installation and fixed maintenance cost for them; which leads to very low price per unit usage in their lifespan. On the other hand, brown energy is derived from off-site electricity that, besides its high per unit usage cost, also leaves carbon dioxide in the environment. We show the brown energy cost for the specific type of VM as $c_{b}$ per unit time and the time duration brown energy has been used while migration takes place as $T_{b}$. Therefore, the cost of additional brown energy usage can be shown as:

$$
C_{\text {addBrown }}=c_{b} \times T_{b} .
$$

\subsection{System Objective}

Considering prices of different energy sources and their environmental impact, running VMs using renewable energy sources eventually leads to a lower total cost. We consider powering data centers using renewable energy unless it is not available. Since renewable energy sources have intermittent nature, there is the possibility of their shortage in the lifetime of a VM running in the data center. In this case, cloud provider could migrate the VM to another availability zone with excess renewable energy available. Performing VM migration could lead to lowering and even eliminating brown energy usage, but it imposes extra costs to the system. In this work, our objective is to minimize the total cost of running VMs in the system through VM migration. As shown in Equation (9), the objective function consists of energy used in data centers to run VMs, and extra energy used to migrate VMs to the data center with access to renewable energy and the additional brown energy used in the source data center while migration takes place.

$$
\text { minimize } C_{\text {execution }}+C_{\text {extra }} \text {. }
$$

The first part (execution cost) in the objective function is inevitable even if no migration takes place. Therefore, to achieve our goal we restate the objective function as to minimize the extra cost 
due to VM migration. However, optimal cost minimization within a data center with very large number of VMs is a complex problem. We narrow down our formulation to a single VM migration problem, which eventually leads to overall cost minimization when the cost for the individual VM is minimized.

\subsection{VM Migration Problem}

To maximize renewable energy usage and be aligned with the system objective, we perform VM migration in the absence of renewable energy. The extra energy, Equation (2), consists of the energy used by the network, Equation (7), and additional brown energy used at the source data center, Equation (8), while the VM migration takes place.

We break down the extra cost into three different cases, as shown in Equation (10) and Figure 2.

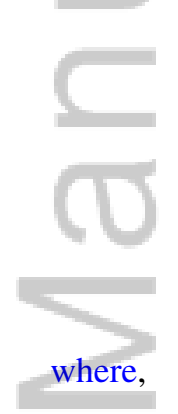

$$
C_{\text {extra }}= \begin{cases}C_{1} & \text { if } t_{m}<t_{b} \text { and } t_{b}-t_{m} \geq T_{m} \\ C_{2} & \text { if } t_{m}<t_{b} \text { and } t_{b}-t_{m}<T_{m} \\ C_{3} & \text { if } t_{m} \geq t_{b} .\end{cases}
$$

$$
\begin{gathered}
C_{1}=c_{n} \cdot T_{m}, \\
C_{2}=C_{3}=c_{n} \cdot T_{m}+c_{b} \cdot\left(t_{m}-t_{b}+T_{m}\right) .
\end{gathered}
$$

The first case $\left(C_{1}\right)$ indicates when the VM migration starts at $t_{m}$ and finishes before the start of brown energy consumption $t_{b}$. This is shown in case (a) in Figure 2 as well and can be formulated as: $t_{m}<t_{b}$ and $t_{b}-t_{m} \geq T_{m}$. Therefore, the time duration required for VM migration to be completed $T_{m}$ comes to at end before the data center starts to use brown energy sources and the only extra cost in this case is the migration cost as shown in Equation (11). $C_{2}$, case (b) of Figure 2, occurs when migration starts before finishing of renewable energy $t_{m}<t_{b}$ but it completes after start of brown energy usage $t_{b}-t_{m}<T_{m}$. As shown in Equation 11, besides the migration cost, the cost of brown energy usage in the source data center is added to the extra cost as well. Finally, $C_{3}$ which is the case (c) in Figure 2, occurs after the time no renewable energy is available in the data center, i.e., $t_{m} \geq t_{b}$. 
Case (a)

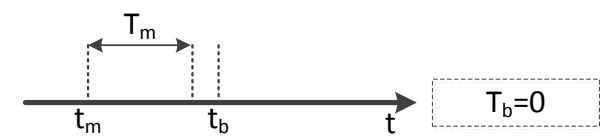

Case (b)

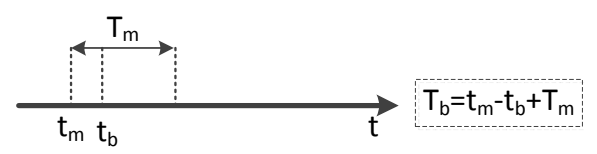

Case (c)

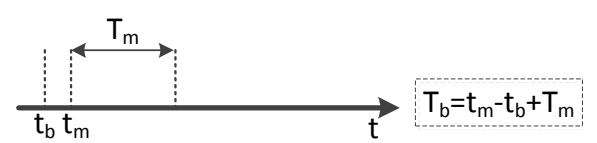

Figure 2. Example of migration time $\left(t_{m}\right)$ versus start time of brown energy consumption $\left(t_{b}\right)$.

\section{OPTIMAL OFFLINE VM MIGRATION}

In this section, we study the offline solution of a single VM migration problem among data center sites to increase the usage of renewable energy sources. Without loss of generality, we assume that the brown energy cost per unit time to be 1 and normalize the network cost $c_{n}$ to the brown energy cost, as shown in Equation (12).

$$
c_{b}=1 \text { and } c_{n}=s ; \text { where } s \in \mathbb{R}^{+} .
$$

Moreover, we consider the following relation for $t_{b}, t_{m}$, and $T_{m}$.

$$
t_{b}-t_{m}=a T_{m} ; \text { where } a \in \mathbb{R} .
$$

Considering Equations (12) and (13), we rewrite Equations (10) and (11) as follows:

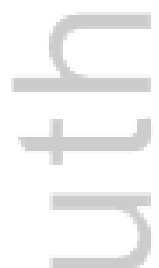

$$
C_{\text {extra }}= \begin{cases}C_{1}=s \cdot T_{m} & \text { if } a \geq 1, \\ C_{2}=C_{3}=s \cdot T_{m}+(1-a) T_{m} & \text { if } a<1 .\end{cases}
$$

\section{Theorem 1}

The optimal offline (OPT) cost is $s \cdot T_{m}$.

\section{Proof}

However, finding the optimal offline cost associated with Equation (14) is straight forward, we provide the detailed proof for better understanding of VM migration problem under different system 
conditions. In order to find the optimal offline solution, we need to find the condition where the cost function has the minimum cost. Based on Equation (14), we have:

1. $C_{1}$ equals $s . T_{m}$, where $a \geq 1$.

2. If $a<1$ then $1-a$ is always a positive value and $C_{2}$ or $C_{3}$ are always greater than $s . T_{m}$, which means $C_{2}>C_{1}$ or $C_{3}>C_{1}$.

As a result, the optimal offline happens at $a \geq 1$ or $t_{b}-t_{m} \geq T_{m}$. This means that the optimal offline happens when migration starts and finishes before the start of brown energy usage in the data center. This leads to the optimal offline cost $s \cdot T_{m}$.

\section{ONLINE VM MIGRATION}

In this section, we construct two online algorithms to minimize cost of VM migration. The reason for proposing online algorithms is that optimal offline algorithm is only attainable when we have full future knowledge about the system and renewable energy level. Here we propose two deterministic online VM migration algorithms: optimal online deterministic (OOD) VM migration with no future knowledge and future-aware dynamic provisioning (FDP) VM migration with limited knowledge (up to a window-size) regarding renewable energy level. Our online algorithms are inspired by skirental problem [44]. We decide when to migrate a VM to another data center with excess renewable energy to minimize brown energy consumption. It should be noted that the decision to whether or not to migrate a VM to another data center is considered to be happening in serial. Making decision to migrate the VMs in this way, we assume that we only make decision regarding migration of one $\mathrm{VM}$ at a time and our knowledge about the renewable energy level at the destined data center is precise to large extent. Moreover, in our model we keep two copies of VM while migration and switching is happening. Keeping a copy of the VM in the source data center till VM migration fully completes assures that user experience in terms of latency and response time would not be affected by the migration time and network delay. 
In order to be able to evaluate the performance of our online algorithms, we use the competitive ratio analysis [45].

\section{Definition}

An online algorithm is called c-competitive if, for all possible inputs, the outcome of the online algorithm $\left(C_{A}\right)$ in comparison to the optimal offline outcome $\left(C_{O P T}\right)$ has the following relation: $C_{A} / C_{O P T} \leq c$

\subsection{Optimal Online Deterministic VM Migration}

Our goal is to propose an algorithm that could achieve optimal result using only the current information available. Theorem 2 shows the optimal online deterministic algorithm for a single VM migration problem is attained when migration takes place by the beginning time of brown energy usage, that is, $t_{m}=t_{b}$.

\section{Theorem 2}

The optimal online deterministic (OOD) algorithm is achieved when $t_{m}=t_{b}$ and it is $(1+1 / s)$ competitive.

Proof

Based on the cost function in Equation (14) and Theorem 1, we can write the competitive ratio for any arbitrary online algorithm with no future knowledge as follows:

$$
\frac{C_{O O D}}{C_{O P T}} \leq \begin{cases}\frac{s \cdot T_{m}}{s \cdot T_{m}}=1 & \text { if } a \geq 1 \\ \frac{s \cdot T_{m}+(1-a) T_{m}}{s \cdot T_{m}}=1+\frac{1-a}{s} & \text { if } a<1 .\end{cases}
$$

where $a=\frac{t_{b}-t_{m}}{T_{m}}$ as defined in Equation (13).

Any online algorithm with no future knowledge can only have the knowledge of the current time $t_{i}$, and $t_{b}$ if $t_{b} \geq t_{i}$, that is, the time from which VM started using brown energy. Accordingly, two different groups of online algorithms with no future knowledge can be defined that they set $t_{m}$ as a function of:

1. the current time $t_{i}$, i.e., $t_{m}=f_{1}\left(t_{i}\right)$, and 
2. the start time of brown energy usage, i.e., $t_{m}=f_{2}\left(t_{b}\right)$.

For algorithms from the former group, $a=\frac{t_{b}-f_{1}\left(t_{i}\right)}{T_{m}}$, since $a$ is not a function of $t_{b}$, $a$ can grow arbitrarily large when the adversary will select $t_{b}$ such that it is infinitely greater than $f\left(t_{i}\right)$, i.e., $a \rightarrow \infty$, and as a result $\frac{C_{O O D}}{C_{O P T}} \rightarrow \infty$. Therefore, all algorithms from the first group are not competitive.

$=$ For algorithms from the latter group, $a=\frac{t_{b}-f_{2}\left(t_{b}\right)}{T_{m}}$, the time of migration $t_{m}$ is dependent to the start time of brown energy usage $t_{b}$ which is known for the algorithm, therefore:

$$
\begin{gathered}
t_{m} \geq t_{b}, \\
\text { as } a=\frac{t_{b}-t_{m}}{T_{m}} \Rightarrow a \leq 0 .
\end{gathered}
$$

Considering $a \leq 0$, the minimum competitive ratio is achieved when $a=0$ for the second inequality in Equation (15). This means migration starts by the beginning of brown energy usage, i.e., $t_{m}=t_{b}$. As a result, the best competitive ratio is $1+\frac{1}{s}$.

\subsection{Future-Aware Dynamic Provisioning VM Migration}

As mentioned earlier, we consider access to renewable energy sources along with the electricity derived from off-site grid. Two renewable sources consider in this paper, solar and wind, have different pattern during the day. As shown in the Figure 4a, solar energy has a predictable pattern during the day and its peak is foreseeable. In contrast, wind energy does not have a predictable diurnal pattern. But one can use the average temporal pattern of wind energy, which can be captured in the region [46]. It is often assumed that the renewable energy availability in the near look-ahead window can be predicted with a good accuracy in reality, such as auto-regressive techniques used in the works by Kansal et al. [47] and Cox [48]. If there are prediction errors in the model, decisions would be affected by the same error margin as prediction errors. For example, 10\% prediction error causes $10 \%$ error in decision making. If the time window is small enough, such as minutely windows, then renewable energy prediction can be predicted with considerably high precision almost similar to real time measuring; therefore, it will not affect the decisions significantly. The 
question is how much knowledge can help and get the online algorithm performance close to the optimal offline algorithm.

We assume that at any given time, $t_{i}$, the future renewable energy is predictable for a window-size $\omega$, which means the amount of renewable energy in the system is known for the period $\left[t_{i}, t_{i}+\omega\right]$. Now we elaborate on how the window-size affects the decision making process and improves the online algorithm performance. The following two cases are plausible:

- If window-size is greater or equal to the time required to perform the migration, $\omega \geq T_{m}$, it would be the same as the scenario for optimal offline algorithm. Therefore, there is enough time to migrate the VM to a data center with access to renewable energy and avoid brown energy usage.

- If window-size is smaller than the time of migration, $\omega<T_{m}$, then $t_{m}+\omega \geq t_{b}$.

\section{Theorem 3}

The competitive ratio for the future-aware dynamic provisioning (FDP) algorithm is: $\frac{C_{F D P}}{C_{O P T}} \leq$ $1+\frac{1}{s}-\frac{\omega}{s \cdot T_{m}}$, where $w \leq T_{m}$.

\section{Proof}

The optimal offline algorithm migrates the VM, $T_{m}$ unit of time earlier than $t_{b}$ and the optimal online deterministic algorithm with no future knowledge migrates the VM by the time of $t_{b}$. The FDP algorithm with limited future knowledge minimizes the cost when migrates the VM as soon as $t_{b}$ is known. That is, FDP can migrate the VM at most up to $\omega$ unit of time earlier, when $t_{b}$ can be seen within the look-ahead window. Therefore, there would be $\omega$ unit less brown energy consumption which improves the online algorithm cost. Equation (17) shows the competitive ratio for future-aware online algorithm.

$$
\frac{C_{F D P}}{C_{O P T}} \leq \frac{s \cdot T_{m}+T_{m}-\omega}{s \cdot T_{m}}=1+\frac{1}{s}-\frac{\omega}{s \cdot T_{m}},
$$

where $w \leq T_{m}$, as competitive ratio is always greater or equal to 1 . 


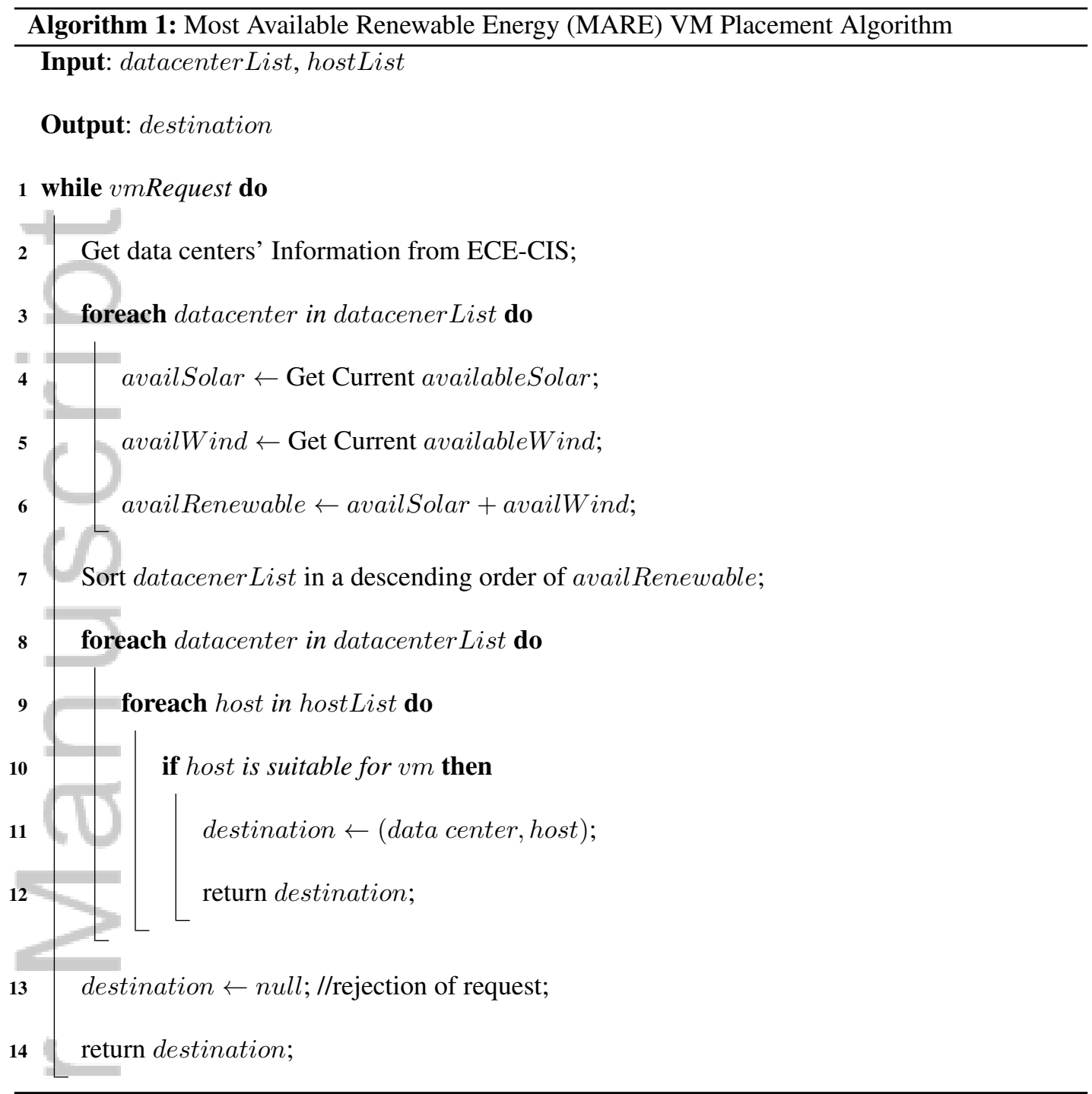

\subsection{VM Placement}

By the arrival of each VM request, the broker should allocate resources to the VM and for this purpose it needs to decide where to place the VM. We treat VM placement as a bin-packing problem with different bin sizes, which are physical servers in this context. Since bin-packing is an NP-hard problem, we use derivation of best-fit heuristic to solve it. To be aligned with our purpose and taking the most from available renewable energy in distributed data centers, we consider a modification of the best-fit heuristic that we proposed in our previous work [38]. The modification to the Energy and Carbon-Efficient (ECE) algorithm [38] is denoted as Most Available Renewable Energy (MARE). 
MARE sorts data center sites according to the amount of available renewable energy and submits the VM to the data center with the highest available amount. The pseudo-code of the VM placement algorithm is presented in Algorithm 1.

The time complexity of Algorithm 1 with $v$ VM requests, $n$ data center sites, and $h$ physical servers within each data center, in detail is as follows: Lines 3-6 take $O(n)$ and the sort function in Line 7 can be done in $O(n \log (n))$. Lines 8-12 take $O(n h)$, in the worst case. Thus, the total running time for the algorithm is $O(v(n+n \log (n)+n h))$. Since the number of VM requests and hosts dominate the total number of data center sites $(n)$, the total time complexity of the algorithm is $O(v n h)$.

In addition, we consider another VM placement algorithm without any knowledge regarding renewable energy availability, denoted as Random algorithm. By the arrival of a new VM request, Random algorithm chooses a random data center uniformly.

\section{PERFORMANCE EVALUATION}

We perform simulation-based experiments to evaluate our proposed algorithms. Our aim is to measure the energy cost savings incurred due to migration of VMs to data centers with access to renewable energy sources. Moreover, we measure the improvement made by applying the proposed approaches in cutting the amount of carbon emission.

Workload Data. We use Google cluster-usage traces [16] for workload as there is no other publicly available real-world workload traces for IaaS cloud providers to the best of our knowledge. The Google dataset has records of one cluster's usage (which is a set of 12,000 physical machines) and includes submitted requests to that cluster over a period of one month. Each request has requirements shown as amount of requested CPU, memory, and storage. Since these traces are user requests not representing VM instances demand, we need to make a mapping between request submissions from users to IaaS computing demand. We use the same technique used by Toosi et al. in [49] as part of their workload generation to generate VM request traces. 


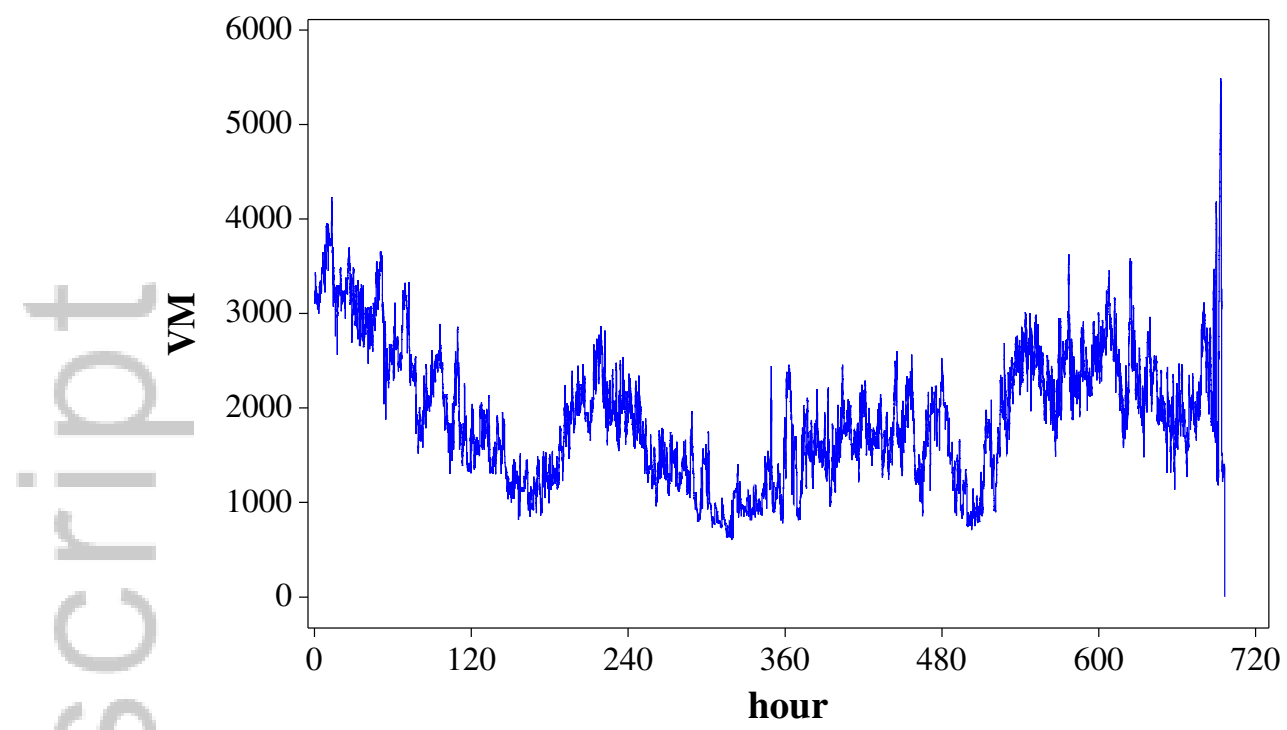

Figure 3. One month Google workload trace.

Google traces include record of users, each submitting several tasks, with specific resource requirements. Considering the fact that $93 \%$ of the Google cluster machines have the same computing capability, we assume all physical machines in the cluster have the same resources (in terms of CPU, memory, etc.) and map our VM size to that of the physical machine. In order to derive VM request traces from Google traces, whenever a user submits a task we check if there is already a VM instantiated by that user in the system with enough computing resources to run the new task. Otherwise, if there is no VM owned by the user with enough capacity to accommodate the new task, we instantiate a new VM to serve the user's task [49]. We also terminate a VM when there is no task running on it. By this, we can create a trace of VM requests submitted from users. The trace contains 250,171 VM requests, each has the start time and holding time in the data center. We consider the VM specifications in our model similar to the standard small instances introduced by Amazon EC2 [13]. Figure 3 shows the number of VM requests per hour received by the provider, generated based on the scheduling algorithm we used to generate VM requests according Google cluster traces. This figure shows the shape of the workload and its fluctuation in our simulations.

Data Centers' Configuration. We consider 3 data center sites located in the US-West region. The locations are chosen from the Data centers Map [50], and are as follows: Phoenix in Arizona, 
Los Angeles in California, and Cedar City in Utah. The number of servers in each data center is set in a way that data centers' capacity would not be a limitation for not being able to take advantage of available renewable energy. Based on the previous discussion, the servers in the data centers are homogeneous with equal processing capacity. We model servers in data centers based on the latest HP ProLiant DL360 Gen9 server [51], with following specifications: Intel Xeon E5-2670v3, 10 cores $\times 2.3 \mathrm{GHz}, 256 \mathrm{~GB}$ memory. The power consumed by each request running on a server within a data center is assumed to be on average a constant rate per time slot (e.g., 550W/hour).

We consider $P U E$ value of 1.4 for all data center sites to calculate the overhead energy usage. The reason is that we aim to evaluate algorithms in a setting where PUE values are not determinative. We select the carbon footprint $=0.350$ Tones $/ M W h$ for the off-site grid electricity, derived from the US Department of Energy Electricity Emission Factors [52]. The electricity price of $p_{b}=6.22$ cents $/ k W h$ is chosen for the off-site electricity from the US Energy Information Administration [53]. This price represents the electricity price for the on-peak period, between 8AM and 10PM. We opt the off-peak price to be half of the on-peak. Moreover, as discussed earlier, we consider a fixed price for renewable energy usage per unit as $p_{r}=1.0$ cents. $k W h$.

Renewable Energy Traces. We use the measurements reported by NREL [15] for irradiance and meteorological data from different stations to capture wind and solar energy with 1-hour granularity from May, $1^{\text {st }}$ to May, $29^{\text {th }}$ 2013. To calculate the output for PV power, we use the hourly solar irradiance reported for flat plates on tilted surface at a 45-degree angle and PV efficiency of 30\%. We calculate the solar output based on [54] and the total area for the flat plates is considered to be $100 \mathrm{~m}^{2}$, derived from the configuration by Solarbayer [55]. To generate hourly wind energy, we use the proposed method by Fripp et al. [46]. The hourly wind speed, air temperature, and air pressure, derived from NREL measurements, are fed to the model and the generated power is computed accordingly, assuming each data center uses a GE $1.5 \mathrm{MW}$ wind turbine. Figures $4 \mathrm{a}$ and $4 \mathrm{~b}$ show the solar and wind energy availability for three different cities in our system model, respectively. 


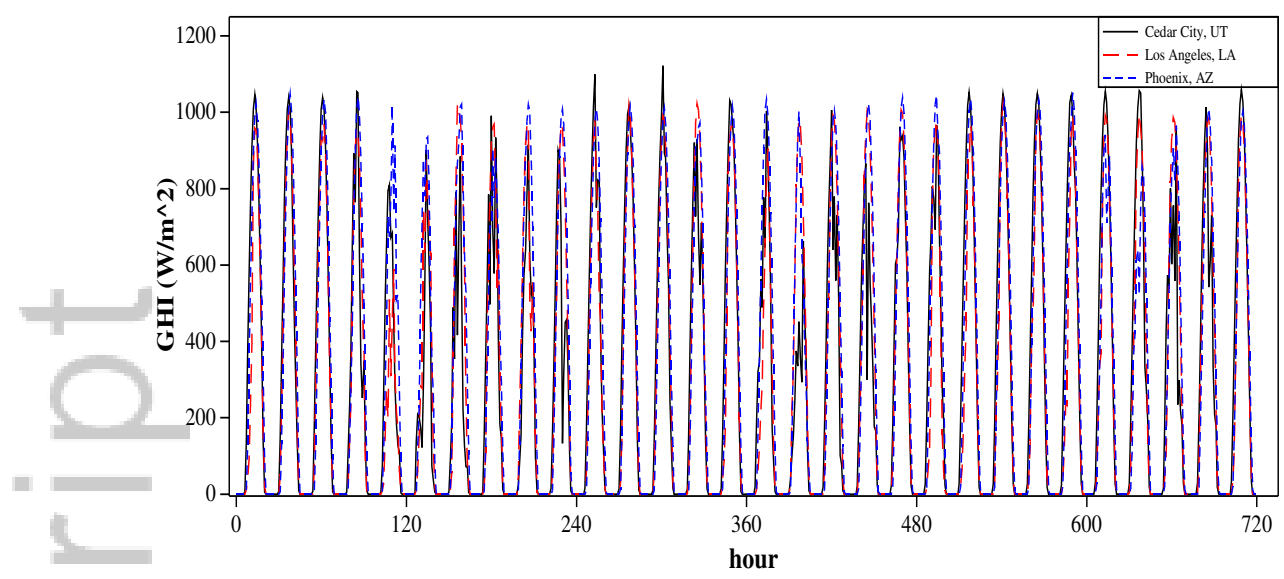

(a) Global horizontal irradiance (GHI).
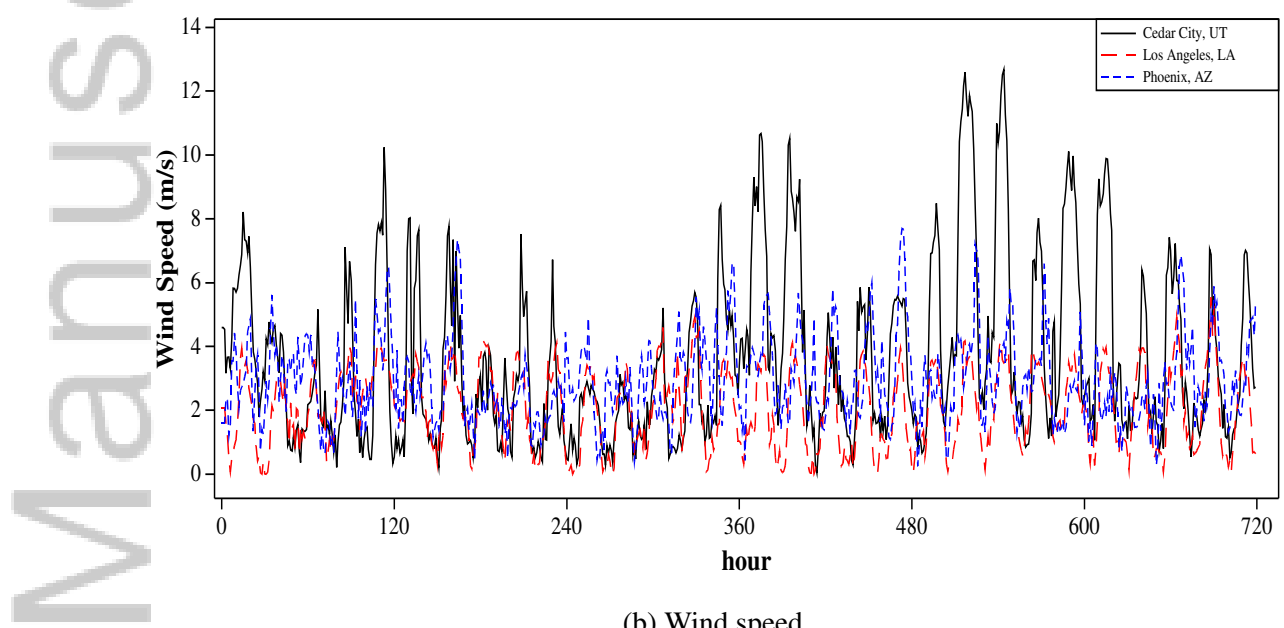

(b) Wind speed.

Figure 4. Renewable energy traces.

\subsection{Experiment Setup}

Benchmark Algorithm. We compare the proposed offline, optimal online, and future-aware algorithms with a baseline benchmark algorithm with no VM migration. The benchmark does not take any further action and does not perform any migration after initial placement and instantiation of the VMs in the data ceners. The benchmark is referred to as No-Migration (NM) policy. 


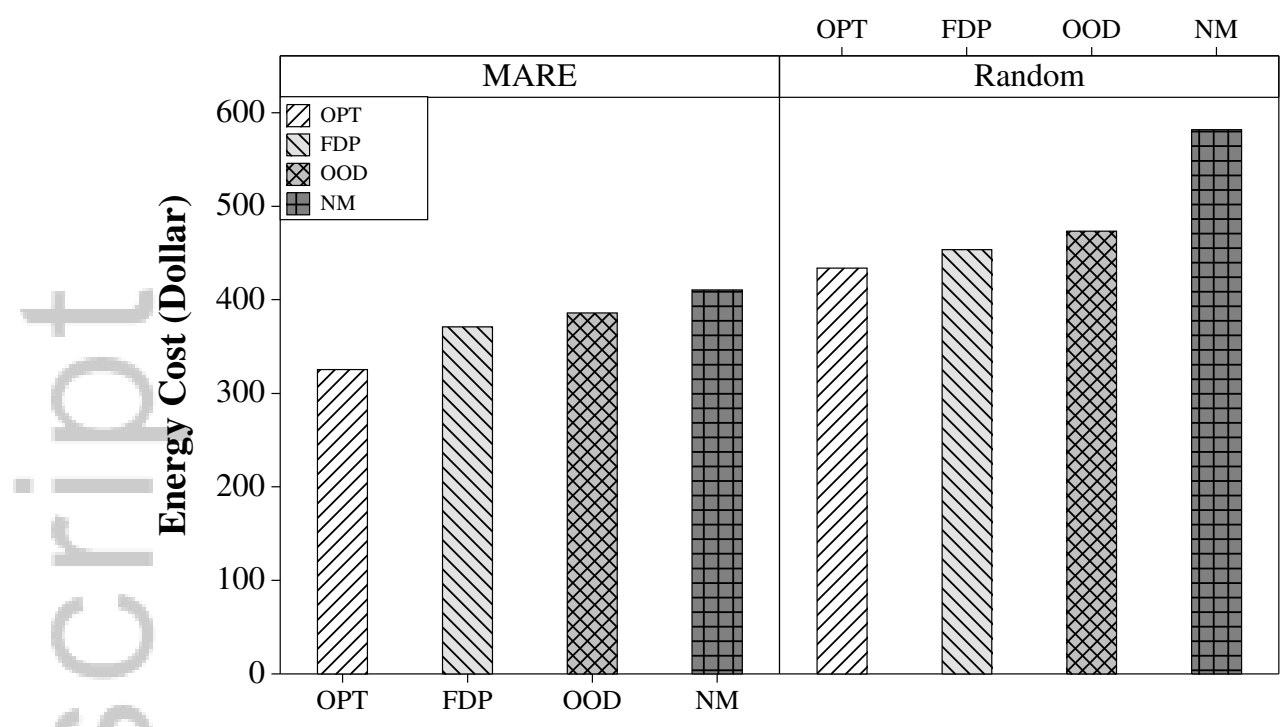

Figure 5. Total energy cost.

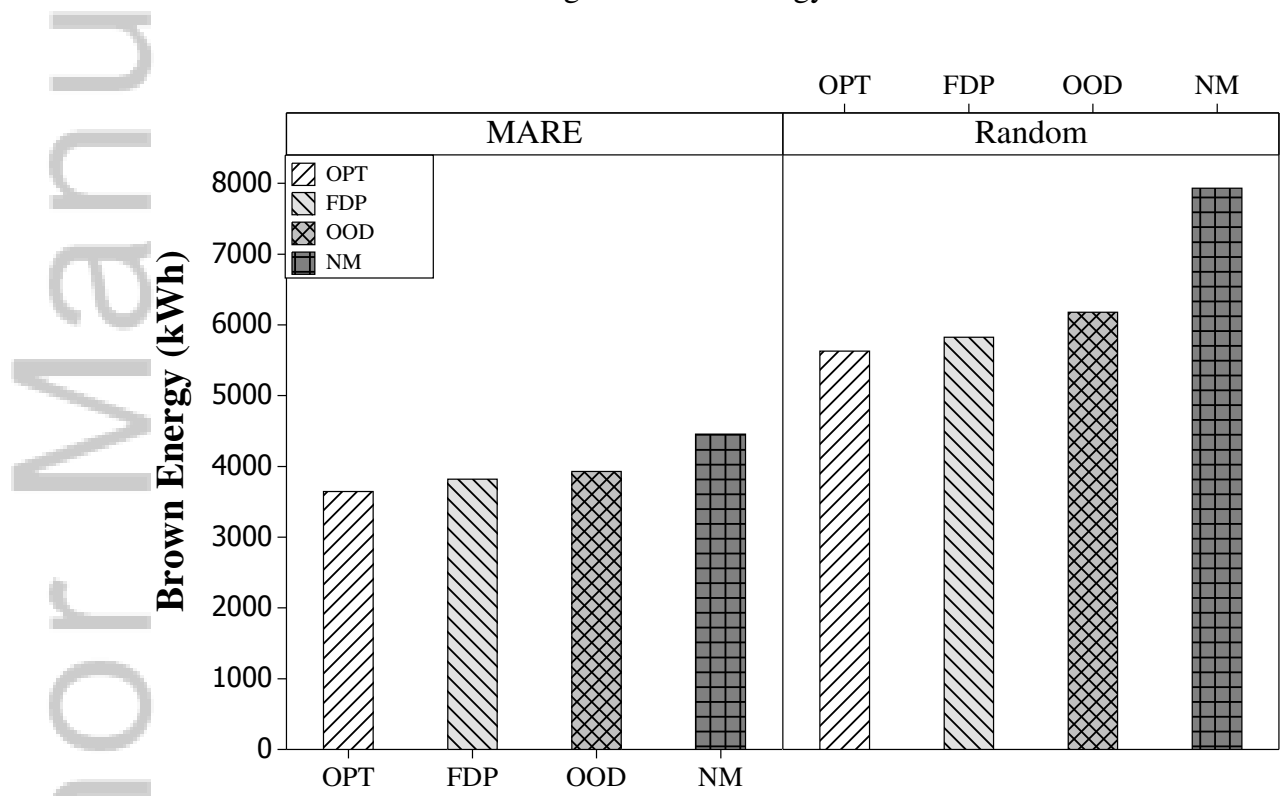

Figure 6. Brown energy consumption.

\subsection{Experiment Results and Analysis}

In the experiments, we use the real-world traces derived from Google to study the performance of the proposed offline, optimal online, and future-aware algorithms all in combination with two VM placement policies against the benchmark algorithm. The results are shown in Figures 5 to 7.

Figure 5 shows the total energy cost incurred by all algorithms, when the window-size for future-aware algorithm is set to 4.5 minutes. The results indicate that having initial knowledge 


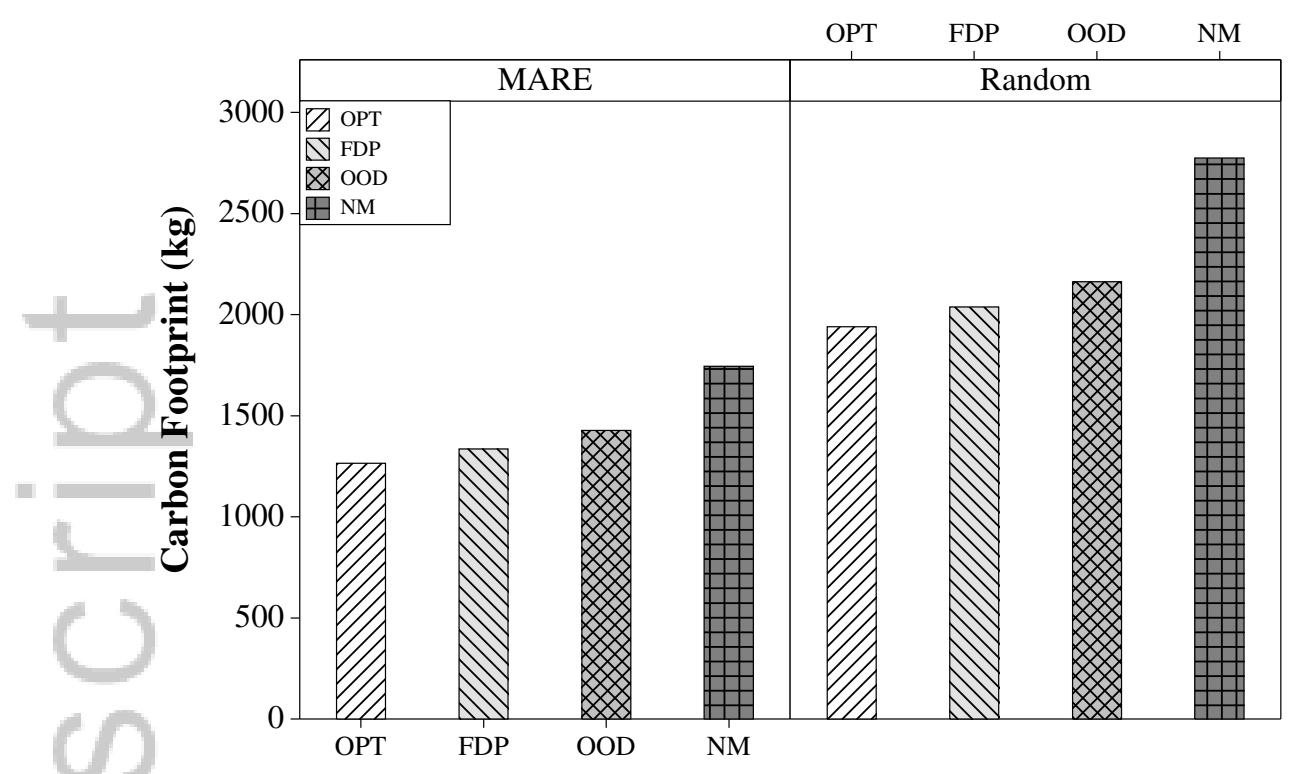

Figure 7. Carbon footprint.

about the current renewable energy level in the data centers has a substantial effect in the amount of cost reduction. It can be seen that there is a significant cost reduction for policies under the MARE placement in comparison to the Random. Since offline policy has the full knowledge of renewable energy in the system, it achieves the lowest cost, $14 \%$ and $18.5 \%$ energy cost reduction in comparison to future-aware and online policies, respectively. Future-aware policy performs slightly better than optimal online algorithm and reduces the total cost by $4 \%$ in comparison to the optimal online policy that makes decision instantly without any future knowledge. The benchmark policy has the highest cost, since after placement of VMs and when there is no renewable energy available in the data center it does not take any further action. The benchmark policy on average consumes $26 \%$ more energy cost in comparison to the optimal offline policy under different VM placement algorithms.

We also measured the amount of brown energy consumption as well as carbon footprint in the system as shown in Figures 6 and 7, respectively. Policies under MARE VM placement achieved considerable reduction in brown energy consumption in comparison to the case when VM placement randomly chooses destined data center. Within each category, offline with full knowledge of renewable energy consumes less brown energy, $5.6 \%$ and $12.9 \%$ less brown energy in comparison 


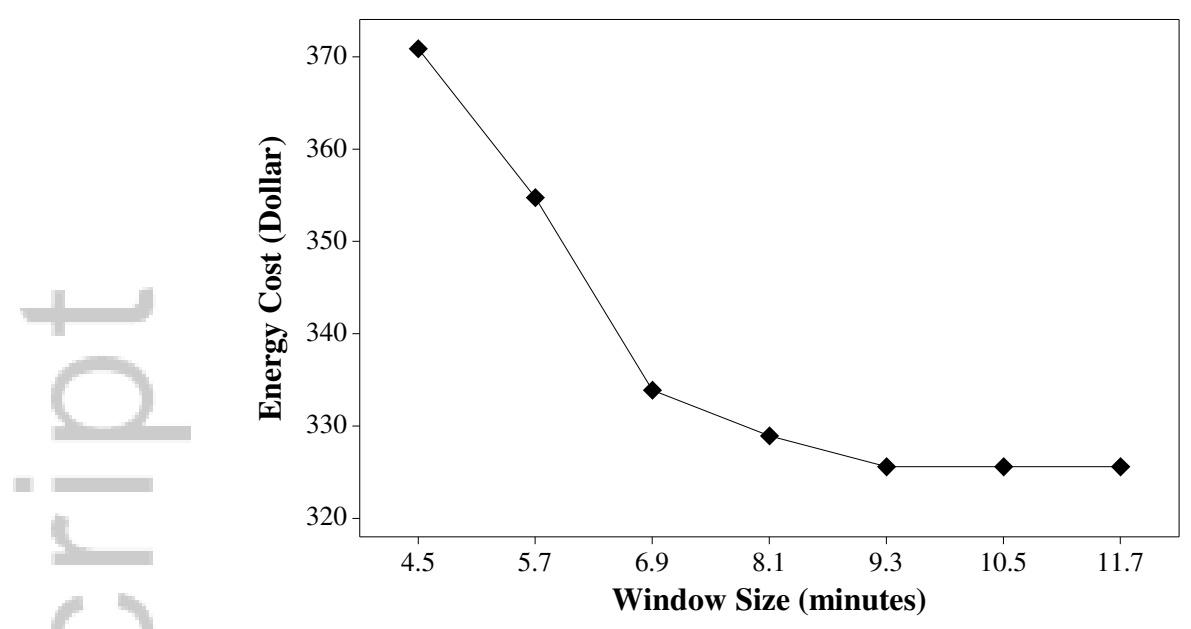

(a) Energy cost.

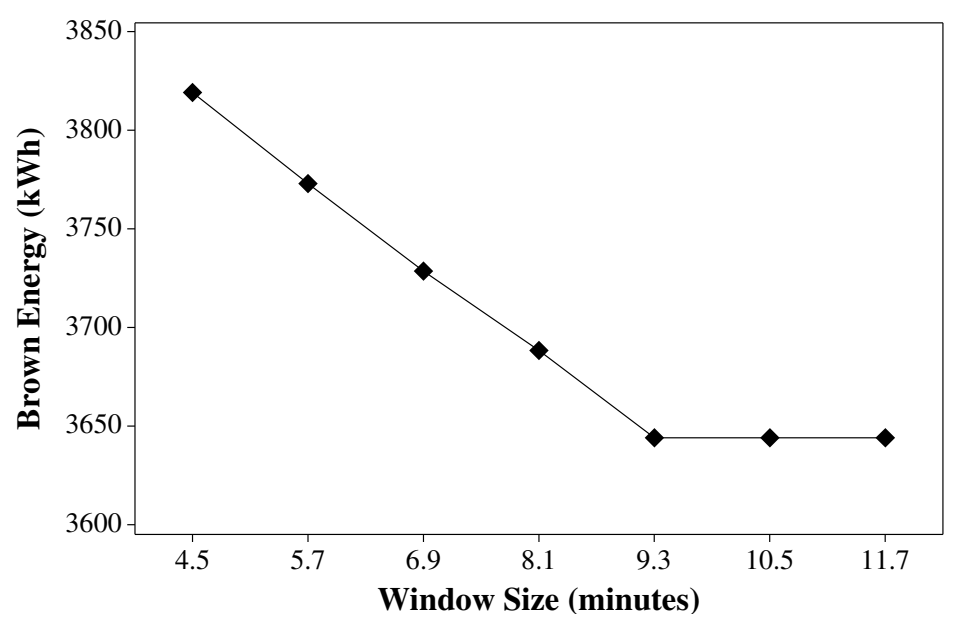

(b) Brown energy consumption.

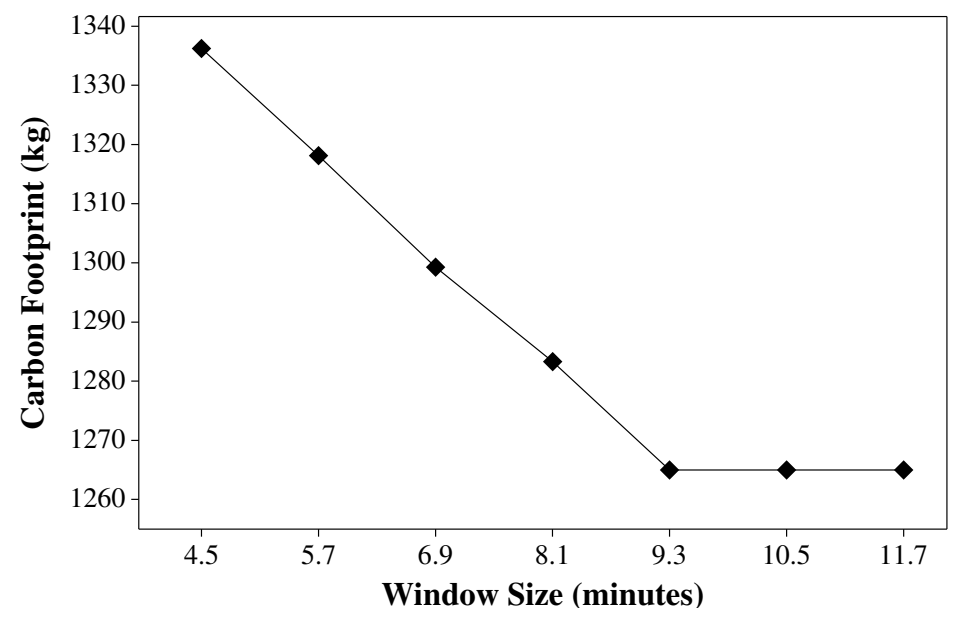

(c) Carbon footprint.

Figure 8. Effect of window-size on the results of future-aware dynamic provisioning algorithm under

MARE VM placement policy. 
to future-aware and online policies, respectively. Future-aware and online policies reduce brown energy consumption by $30.5 \%$ and $22 \%$, respectively, in comparison to the benchmark with no migration. The same behavior can be seen for carbon footprint in Figure 7, since reduction in brown energy consumption eventually leads to lower carbon footprint.

As shown, future-aware policy achieves results that fall between the outcome of the offline algorithm with full knowledge, and optimal online with no knowledge about future renewable energy level. We change the window-size to see its impact on the performance of the future-aware dynamic processioning algorithm. As Figure 8 illustrates, increase in the window-size reduces total cost, brown energy consumption, and carbon footprint. Increase in the window-size makes future-aware algorithm closer to its offline competitor. The performance of the future-aware policy improves and gets close to the optimal offline until window-size reaches 9 minutes. After this point no improvement is achieved, since this is the point that window-size reaches the VM migration time in our experiments. This supports the theoretically proven supposition in Section 1 that if enough knowledge of future is available, the optimal decision suggests a VM migration that finishes before the start of brown energy usage in the data center.

As per Figure 5, the cost ratio of deterministic and future-aware online policies versus the optimal offline algorithm are 1.18 and 1.13, respectively. Moreover, based on the simulation setup $s=3.5$, which leads to deterministic and future-aware online algorithms be 1.28 and 1.14 competitive in comparison to the optimal offline algorithm, respectively. The simulation results are compatible with the calculated competitive ratio as per the provided definition of c-competitive in Section 5.

Figure 9 depicts the total number of migrations happening in the system for each policy during the one-month simulation period and total of $250,171 \mathrm{VM}$ requests. We observe that migration policies under MARE placement achieve lower number of migrations in comparison to the same migration policies under Random placement. The reason is that under MARE placement, a wise data center selection is made for initial VM request placement which reduces the need for possible future migrations. Amongst three different migration policies, offline has the highest number of VM migrations. Since it has full knowledge of the amount of renewable energy in the system and 


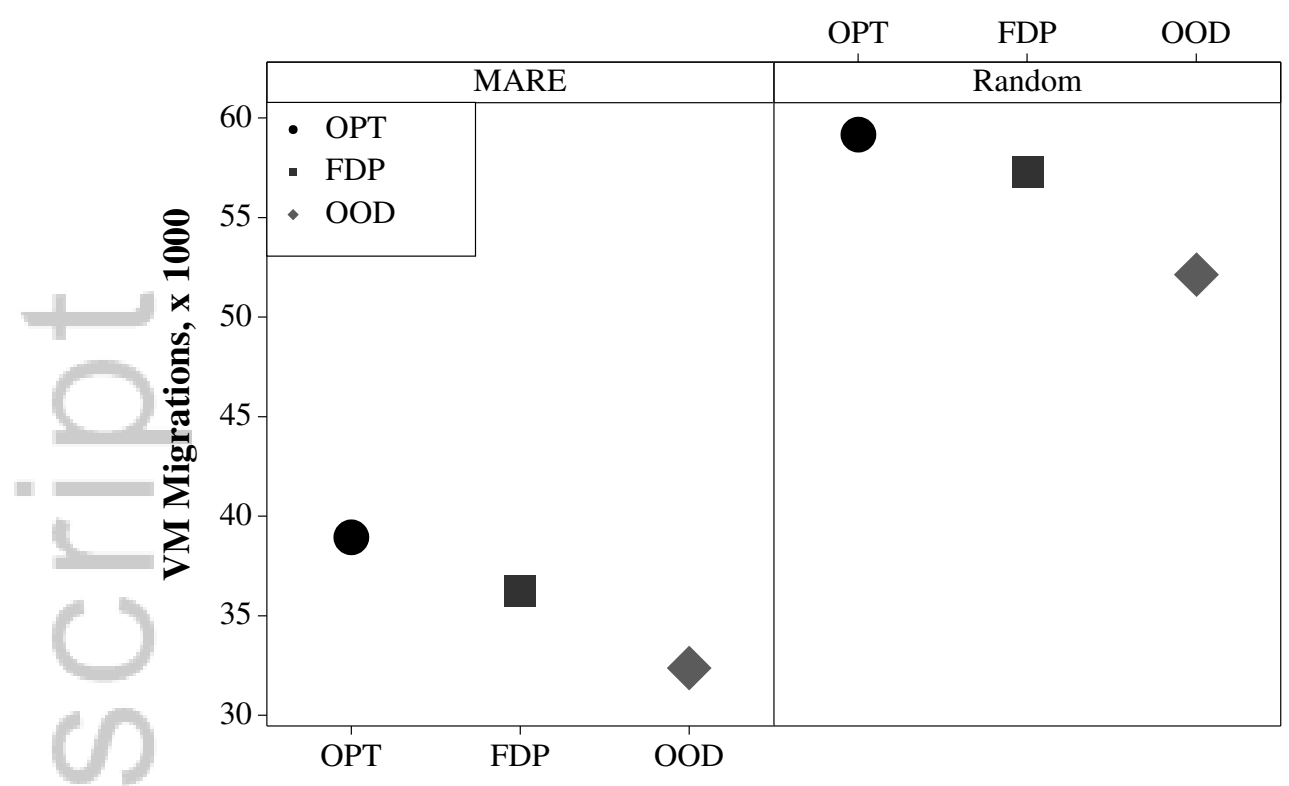

Figure 9. Number of VM migrations.

begins to migrate the VMs before the start time of brown energy usage, unless there is no renewable available in other data centers. Similarly, future-aware policy makes more VM migrations than online policy, due to further knowledge regarding renewable energy level.

\section{CONCLUSIONS AND FUTURE DIRECTIONS}

Using on-site renewable energy sources instead of electricity derived from off-site grid helps cloud providers reduce their energy cost and their reliance on polluting energy sources. Since the nature of renewable energy sources (solar/wind) is intermittent, we take advantage of having access to several geographically distributed data center sites of a cloud provider to perform intra-region VM migration and take the most of the available renewable energy. In this paper, we introduced algorithms with full and partial knowledge of future availability of renewable energy levels to migrate the VMs to another data center within a region in the absence of renewable at the host data center. We first introduced the optimal offline algorithm to minimize the energy cost. Due to the necessity of having full knowledge of future level of renewable energy for optimal offline, we propose two online algorithms. The first online algorithm is a deterministic algorithm that does not 
have any knowledge regarding the future level of renewable energy and the second one is denoted as future-aware online algorithm with limited knowledge, up to a window-size $(\omega)$, of future level of renewable energy. We have compared the results of the proposed optimal offline, optimal online, and future-aware algorithms with a basic benchmark algorithm, that does not perform any migration, all in combination with two VM placement algorithms. One VM placement is aware of the current renewable level, known as MARE, and the other one randomly chooses the destined data center. $=$

We have evaluated the proposed algorithms through extensive simulations using real-world traces for renewable energy (solar and wind) and one-month workload trace of a Google cluster usage. The offline algorithm with full knowledge of renewable energy level performs the best in comparison to the future-aware and optimal online algorithms. The optimal online algorithm incurs $18.5 \%$ more cost compared to the offline algorithm when no future knowledge is available. Moreover, simulation results show that future-aware algorithm's performance gets competitive with offline algorithm by the increase in its window-size until the window-size reaches the network delay or the time needed that a migration takes place and gets completed.

As a future direction, one can study the effect of inter-region migrating of VMs to evaluate the improvements in energy cost versus network delay. Moreover, studying selection of VMs to perform VM migration is another issue that could be investigated; since there are situations that VM migration could lead to violating service level agreement of some users' with special requirements or VM migration needs large amount of data transfer over the network due to data unavailability in the destination. Another important topic of future research is considering a more complex problem, which involves the migration of multiple VMs, the effect of sharing the network on the transfer time, and evaluating the competitiveness of the possible online algorithms in comparison to the optimal offline algorithm.

REFERENCES

1. Armbrust M, Fox A, Griffith R, Joseph AD, Katz R, Konwinski A, Lee G, Patterson D, Rabkin A, Stoica I, et al.. A view of cloud computing. Communications of the ACM 2010; 53(4):50-58. 
2. Brown R, et al.. Report to congress on server and data center energy efficiency: Public law 109-431. Lawrence Berkeley National Laboratory 2008; .

3. Koomey JG. Worldwide electricity used in data centers. Environmental Research Letters 2008; 3(034008).

4. Hamilton J. Cooperative expendable micro-slice servers (cems): low cost, low power servers for internet-scale services. Proc. of the Conference on Innovative Data Systems Research (CIDR), 2009.

5. Mankoff J, Kravets R, Blevis E. Some computer science issues in creating a sustainable world. IEEE Computer 2008; 41(8):102-105.

=6. Smith JW, Sommerville I. Green cloud: A literature review of energy-aware computing. University of St Andrews 2010;

7. Facebook Installs Solar Panels at New Data Center. http://www.datacenterknowledge.com/ archives/2011/04/16/facebook-installs-solar-panels-at-new-data-center/.

8. Facebook in Fort Worth: Our newest data center. https://code.facebook.com/posts/ 1014459531921764 /facebook-in-fort-worth-our-newest-data-center/.

9. AWS and Sustainable Energy. http://aws. amazon.com/about-aws/sustainable-energy/.

10. Apple and the Environment. http://www. apple.com/environment/.

11. Renewable energy. http://www.google.com/green/energy/.

12. Microsoft To Use Solar Panels in New Data Center. http://www.datacenterknowledge.com/ archives/2008/09/24/microsoft-uses-solar-panels-in-new-data-center/.

13. Amazon Web Services. http://aws. amazon.com/.

14. Calheiros R, Ranjan R, Beloglazov A, De Rose C, Buyya R. Cloudsim: a toolkit for modeling and simulation of cloud computing environments and evaluation of resource provisioning algorithms. Software: Practice and Experience 2011; 41(1):23-50.

15. Measurement and Instrumentation Data Center (MIDC). http://www. nrel.gov/midc/.

16. Reiss C, Wilkes J, Hellerstein JL. Google cluster-usage traces: format+ schema. Google Inc., White Paper 2011; .

17. Wind-Powered Data Center in Wyoming. http://www.datacenterknowledge.com/archives/ 2007/11/29/wind-powered-data-center-in-wyoming/.

18. Wu CM, Chang RS, Chan HY. A green energy-efficient scheduling algorithm using the dvfs technique for cloud datacenters. Future Generation Computer Systems 2014; 37:141-147.

19. Brey T, Lamers L. Using virtualization to improve data center efficiency. The Green Grid, Whitepaper 2009; 19.

20. Srikantaiah S, Kansal A, Zhao F. Energy aware consolidation for cloud computing. Proc. of the Conference on Power Aware Computing and Systems. USENIX Association, 2008; 10-10.

21. Harney E, Goasguen S, Martin J, Murphy M, Westall M. The efficacy of live virtual machine migrations over the internet. Proc. of the 2nd International Workshop on Virtualization Technology in Distributed Computing. ACM, $2007 ; 1-7$.

22. Beloglazov A, Buyya R, Lee YC, Zomaya A. A taxonomy and survey of energy-efficient data centers and cloud computing systems. Advances in Computers 2011; 82(2):47-111. 
23. Beloglazov A, Buyya R. Optimal online deterministic algorithms and adaptive heuristics for energy and performance efficient dynamic consolidation of virtual machines in cloud data centers. Concurrency and Computation: Practice and Experience 2012; 24(13):1397-1420.

24. Apple Plans 20MW of Solar Power for iDataCenter. http://www.datacenterknowledge.com/ archives/2012/02/20/apple-plans-20mw-of-solar-power-for-idatacenter/.

25. Kong F, Liu X. A survey on green-energy-aware power management for datacenters. ACM Computing Surveys (CSUR) 2014; 47(2):30.

26. Le K, Bianchini R, Martonosi M, Nguyen T. Cost-and energy-aware load distribution across data centers. Proc. of Workshop on Power-Aware Computing and Systems (HotPower), 2009; 1-5.

27. Liu Z, Lin M, Wierman A, Low SH, Andrew LL. Geographical load balancing with renewables. ACM SIGMETRICS Performance Evaluation Review 2011; 39(3):62-66.

28. Lin M, Liu Z, Wierman A, Andrew LL. Online algorithms for geographical load balancing. Proc. of the International Green Computing Conference (IGCC), IEEE, 2012; 1-10.

29. Toosi AN, Buyya R. A fuzzy logic-based controller for cost and energy efficient load balancing in geo-distributed data centers. Proc. of the 8th IEEE/ACM International Conference on Utility and Cloud Computing (UCC), 2015.

30. Rao L, Liu X, Xie L, Liu W. Minimizing electricity cost: optimization of distributed internet data centers in a multi-electricity-market environment. Proc. of INFOCOM, IEEE, 2010; 1-9.

31. Ren S, He Y, Xu F. Provably-efficient job scheduling for energy and fairness in geographically distributed data centers. Proc. of the 32nd International Conference on Distributed Computing Systems (ICDCS), IEEE, 2012; 2231.

32. Buchbinder N, Jain N, Menache I. Online job-migration for reducing the electricity bill in the cloud. Proc. of the International IFIP TC 6 Networking Conference. Springer, 2011; 172-185.

33. Chen C, He B, Tang X. Green-aware workload scheduling in geographically distributed data centers. Proc. of the IEEE International Conference on Cloud Computing Technology and Science (CloudCom), 2012; 82-89.

34. Celesti A, Puliafito A, Tusa F, Villari M. Energy sustainability in cooperating clouds. Proc. of the 3rd International Conference on Cloud Computing and Services Science (CLOSER), 2013; 83-89.

35. Le K, Bianchini R, Nguyen TD, Bilgir O, Martonosi M. Capping the brown energy consumption of internet services at low cost. Proc. of the International Green Computing Conference, IEEE, 2010; 3-14.

36. Luo J, Rao L, Liu X. Spatio-temporal load balancing for energy cost optimization in distributed internet data centers. IEEE Transactions on Cloud Computing 2015; 3(3):387-397.

37. Le K, Bilgir O, Bianchini R, Martonosi M, Nguyen TD. Managing the cost, energy consumption, and carbon footprint of internet services. ACM SIGMETRICS Performance Evaluation Review 2010; 38(1):357-358.

38. Khosravi A, Garg SK, Buyya R. Energy and carbon-efficient placement of virtual machines in distributed cloud data centers. Proc. of Euro-Par 2013 Parallel Processing. Springer, 2013; 317-328.

39. Belady C, Rawson A, Pfleuger J, Cader T. Green grid data center power efficiency metrics: Pue and dcie. Technical Report, Technical report, Green Grid 2008. 
40. Minas L, Ellison B. Energy efficiency for information technology: How to reduce power consumption in servers and data centers. Intel Press, 2009.

41. Blackburn M, Grid G. Five ways to reduce data center server power consumption. The Green Grid, 2008.

42. Pelley S, Meisner D, Wenisch TF, VanGilder JW. Understanding and abstracting total data center power. Proc. of the Workshop on Energy-Efficient Design, 2009.

43. Clark C, Fraser K, Hand S, Hansen JG, Jul E, Limpach C, Pratt I, Warfield A. Live migration of virtual machines. Proc. of the 2nd Symposium on Networked Systems Design \& Implementation-Volume 2, USENIX, 2005; $273-286$.

44. Karlin AR, Manasse MS, Rudolph L, Sleator DD. Competitive snoopy caching. Algorithmica 1988; 3(1-4):79-119.

45. Borodin A, El-Yaniv R. Online computation and competitive analysis. Cambridge University Press, New York, 2005.

46. Fripp M, Wiser RH. Effects of temporal wind patterns on the value of wind-generated electricity in california and the northwest. IEEE Transactions on Power Systems 2008; 23(2):477-485.

47. Kansal A, Hsu J, Zahedi S, Srivastava MB. Power management in energy harvesting sensor networks. ACM Transactions on Embedded Computing Systems (TECS) 2007; 6(4):32.

48. Cox DR. Prediction by exponentially weighted moving averages and related methods. Journal of the Royal Statistical Society. Series B (Methodological) 1961; :414-422.

49. Toosi AN, Vanmechelen K, Ramamohanarao K, Buyya R. Revenue maximization with optimal capacity control in infrastructure as a service cloud markets. IEEE Transactions on Cloud Computing 2015; 3(3):261-274.

50. Data center map. http://www. datacentermap.com/.

51. HP ProLiant DL360 Generation9 (Gen9). http://h20195.www2.hp.com/v2/gethtml.aspx? docname=c04375623.

52. US Department of Energy, Appendix F, Electricity Emission Factors. http://www.eia.doe.gov/oiaf/

1605/pdf/Appendix20F_r071023.pdf.

53. EIA-electricity data. http://www.eia.gov/electricity/monthly/pdf/epm.pdf.

54. Photovoltaic Education Network. http://pveducation.org/.

55. Solarbayer. http://www. solarbayer.com/.
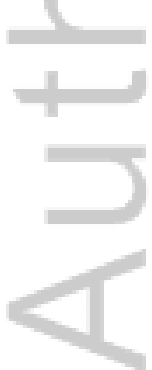


\section{University Library}

\section{- MINERVA \\ A gateway to Melbourne's research publications}

Minerva Access is the Institutional Repository of The University of Melbourne

Author/s:

Khosravi, A;Toosi, AN;Buyya, R

Title:

Online virtual machine migration for renewable energy usage maximization in geographically distributed cloud datacenters

Date:

2017-09-25

\section{Citation:}

Khosravi, A., Toosi, A. N. \& Buyya, R. (2017). Online virtual machine migration for renewable energy usage maximization in geographically distributed cloud datacenters. CONCURRENCY AND COMPUTATION-PRACTICE \& EXPERIENCE, 29 (18), https:// doi.org/10.1002/cpe.4125.

Persistent Link:

http://hdl.handle.net/11343/292902 